Published in Psychosomatic Medicine, Volume 71, Issue 9, pp. 981 - 986, by Lippincott, Williams \& Wilkins / American Psychosomatic Society.

This is a non-final version of an article published in final form in Psychosomatic Medicine, Volume 71, Issue 9, November/December 2009, pp. 981 - 986. Available from: http://hdl.handle.net/1893/1906

Copyright (c) 2009 by the American Psychosomatic Society. 


\title{
A Taxometric Analysis of Type-D Personality
}

\author{
Eamonn Ferguson ${ }^{1} \mathrm{PhD}$, Lynn Williams ${ }^{2} \mathrm{PhD}$, Rory C. O’Connor ${ }^{2} \mathrm{PhD}$, Siobhán \\ Howard $^{3} \mathrm{PhD}$, Brian M. Hughes ${ }^{3} \mathrm{PhD}$, Derek W. Johnston ${ }^{4} \mathrm{PhD}$, Julia L. Allan ${ }^{4} \mathrm{PhD}$, \\ Daryl B. O'Connor ${ }^{5} \mathrm{PhD}$, Christopher A. Lewis ${ }^{6}$ DPhil, Madeleine A. Grealy ${ }^{7} \mathrm{PhD}$ \\ and Ronan E. O'Carroll ${ }^{2} \mathrm{PhD}$
}

${ }^{1}$ School of Psychology, University of Nottingham, Nottingham, UK

${ }^{2}$ Department of Psychology, University of Stirling, Stirling, UK

${ }^{3}$ School of Psychology, National University of Ireland, Galway, Ireland

${ }^{4}$ School of Psychology, University of Aberdeen, Aberdeen, UK

${ }^{5}$ Institute of Psychological Sciences, University of Leeds, Leeds, UK

${ }^{6}$ School of Psychology, University of Ulster at Magee College, Londonderry,

Northern Ireland

${ }^{7}$ Department of Psychology, University of Strathclyde, Glasgow, UK

Correspondence: Eamonn Ferguson, Risk Analysis, Social Processes and Health (RASPH) group, School of Psychology, University of Nottingham, Nottingham, NG7 2RD, UK. Tel: + 44 (0) 115 9515327. Fax: + 44 (0) 115 9515324. Email:

eamonn.ferguson@,nottingham.ac.uk

Short Title: Taxometrics of Type-D

Word $=5,092$

Tables $=2$

Figures $=1$

Support: There was no financial support for this project 


\begin{abstract}
Background: Type-D personality is treated as a categorical variable and caseness has been shown to be a risk factor for poor prognosis in coronary heart disease. However, at present there is no direct evidence to support the assumption that Type-D is categorical and able to differentiate true cases from non-cases.

Methods: In total, 1012 healthy young adults from across the UK and Ireland completed the DS14, the standard index of Type-D, and scores were submitted to two taxometric procedures MAMBAC and MAXCOV.

Results: Graphical representations (comparing actual to simulated data) and fit indices indicated that Type-D is more accurately represented as a dimensional rather than categorical construct.
\end{abstract}

Conclusion: Type-D is better represented as a dimensional construct. Implications for theory development and clinical practice with respect to Type-D are examined as well as the wider use of taxometrics within psychosomatic medicine (e.g., to investigate if there are medically unexplained syndrome taxons such as a Gulf War Syndrome taxon).

Key Words: Type-D, Taxometric Analysis, Taxon, Dimensional, Functional Syndromes

Acronyms: $\mathrm{CHD}=$ Coronary Heart Disease, $\mathrm{IRT}=$ item response theory, $\mathrm{NA}=$ Negative Affectivity, SI = Social Inhibition, $\mathrm{MAMBAC}=$ mean above minus below a cut, $\mathrm{MAXCOV}=$ Maximum Covariance, $\mathrm{MAXEIG}=$ maximum eigenvalue, $\mathrm{L}-$ MODE $=$ L-mode factor analysis, $\mathrm{CCFI}=$ curve comparison fit index, GCMT = General Covariane Mixture Theorem, CFS $=$ Chronic fatigue syndrome. IBS = Irritable bowel syndrome, GWS = Gulf war syndrome. 
Taxometrics of Type-D

The Type-D or 'distressed' personality is defined as the '...tendency to experience negative emotions and to inhibit self-expression' (p 970) [1]. Type D plays an important role in contemporary research on the relationship between personality characteristics and cardiovascular disease [1-5]. Coronary heart disease (CHD) patients classified as Type-D cases have a significantly poorer prognosis [1, 4] leading Type-D to be defined in the literature as a categorical risk factor in CHD. Whether or not initially intended, this implies a qualitative difference between Type-D cases and non-cases. Indeed, the researchers who originally developed the Type-D construct, Denollet and colleagues have, in one paper, directly referred to it as a taxonomy [6], but in other papers they have referred to it as a 'tendency'. This notion of 'tendency' implies dimensionality and indeed others have described Type-D as consisting of ' ... negative affectivity (NA) and social inhibition (SI) personality dimensions' (p 235) [7]. Whether Type-D is dimensional (i.e., distributed as a continuous variable, with individuals varying quantitatively from each other) or taxonic (i.e., individuals are differentiated into non-arbitrary groups or categories) has important clinical and theoretical implications [8]. It has been argued that to explain dimensionality the existence of multiple, additive causal factors that sum to produce quantitative variation on the trait is the most plausible model [8]. This implies that clinicians and researchers should utilize the full range of scores rather than the use of arbitrary cutoffs [cf. 9] and the use of the full range of scores in research [cf. 10] ${ }^{1}$. By contrast, for a categorical approach there is a need to explain the discontinuity between people. This suggests a greater number of possibilities, including a single causal factor (e.g., genetic or threshold models) leading to a dichotomous outcomes or more complex interacting systems such a environmental influences leading to developmental

\footnotetext{
${ }^{1}$ While this should be generally true, a single cause is always a possibility.
} 
Taxometrics of Type-D

bifurcation [8]. While exact mechanisms may not be clear at present the underlying principle is that the existence of dimensional and taxonic models requires very different theoretical accounts. Therefore, it is essential to test directly if Type-D is taxonic or dimensional and this is the focus of this paper.

\section{Evidence for the Dimensionality or Taxonicity Type-D}

Recently, psychometric work using item response theory (IRT) was interpreted as support for a categorical interpretation of Type-D using a cutoff score of 10 on each of the two sub-scales (NA and SI) of the DS14 [11]. This interpretation should be reconsidered. First, it has been argued that, mathematically, IRT methods produce unidimensional factors and so lack the specificity necessary to determine non-arbitrary cutoffs [12-13]. Second, this study only considered a single cutoff of 10 on each sub-scale. There is no way to know if this is the optimal cutoff for achieving validity, or if a more reliable function may have been observed at other cutoffs. More importantly, it should be noted that the original decision to define these cutoffs for Type-D was based on the combination of cluster analysis and the use of median splits rather than on theoretical considerations $[4,14]$.Cluster analysis is limited when it comes to identifying taxons, as (1) it always produces sub-groups, and yet (2) there is no way to establish the appropriate number of sub-groups [15]. Using median splits to identify Type-D cases amounts essentially to using arbitrary cutoffs which others have argued against as a basis for suggesting taxonicity [cf. 9].

The other type of evidence that could be offered to suggest that Type-D is taxonic is based on the following argument: Using these cutoffs Type-D demonstrates good prognostic outcomes. There is a large body of evidence showing that Type-D cases differ from non-cases in terms of mortality [4], morbidity [1], biological markers [16-18] physiological stress responses [19] and psychosocial factors [5, 20]. 
Taxometrics of Type-D

While this evidence appears compelling, it does not demonstrate that Type-D is taxonic, just that there are between-group differences based on median splits. Indeed, it is possible to show that median splits for known dimensional traits (e.g., neuroticism, health anxiety) exhibit differences on numerous outcomes (memory effects, reaction times) $[8,10,21]$. This does not mean that these traits are taxonic. The above evidence that Type-D is categorical is, therefore, weak.

Furthermore, even if there is a Type-D taxon, but the current cutoff is incorrectly positioned and the Type-D non-case category includes cases, then key psychobiological differences may be obscured, at-risk cases missed, and prognostic power reduced. Conversely, if the Type-D case category includes non-cases, biological differences may be obscured and prognostic power would be reduced, although Type-D cases would not be missed.

The idea that Type-D is a dimensional construct comes from the finding that a number of individual differences theoretically and empirically related to the subcomponents of Type-D - worry, depression, anxiety - [14, 22-23] are dimensional [8]. This implies that the latent structure of the sub-components of Type-D, NA and SI, should be dimensional. Thus while indirect, this evidence is based on taxometric evidence and it is reasonable to hypothesize that Type-D may be dimensional.

The Present Study

At present there is no direct evidence concerning whether or not Type-D is dimensional or taxonic. However, even in the absence of direct evidence that Type-D is taxonic, the 'established cutoffs' are still used to split samples into cases and noncases. Given the very different implications for theory, research and clinical practice afforded by dimensional and taxonic conceptualizations, a direct test of the dimensionality of Type D is urgently required [8]. This paper tests the dimensionality 
Taxometrics of Type-D

of Type-D using taxometric procedures [13, 24-26] in an unselected, healthy, young adult sample of university students. We deliberately avoided recruiting a sample containing sub-populations with particular disorders (in this case cardiovascular disease) alongside groups of healthy persons, as such a combination of heterogeneous groups could lead to the erroneous identification of a taxon (i.e., a pseudo-taxon) [8, 15, 26-28]. Indeed in the case of Type-D the evidence shows that the prevalence of Type-D is significantly higher in clinical groups with cardiovascular disease compared to healthy controls [14]. This increased prevalence of Type-D cases in clinical samples may lead to the identification of a pseudo-taxon if combined with a healthy sample where the prevalence rate is significantly lower. Furthermore, a sample of healthy participants is likely to cover the full range of scores on Type-D and be normally distributed limiting skew. Positive skew is known to lead to the identification of pseudo-taxa with low base rates, whereas negative skew can misidentify a high base-rate taxon [see 29]. Furthermore, the initial analysis of the DS14 [14] by Denollet confirmed that the same factor structure was present in both cardiovascular non-clinical and clinical samples. As such, the use of a non-clinical sample in this study is appropriate and consistent with other psychometric analyses [14].

\section{Method}

\section{Sample and Sampling}

In total, 1012 healthy young adults took part in this study (787 females, 225 males: mean age $=20.5$ years, $\mathrm{SD}=4.84$; ages range $17-61$ ). These were recruited via convenience sampling from eight universities across England, Scotland, Northern Ireland and Republic of Ireland. The samples from England, Scotland, Northern Ireland and Republic of Ireland did not differ significantly in terms of age, $(F(5$, 
Taxometrics of Type-D

$1006)=0.740, p=.59)$, or gender $\left(\chi^{2}(6, \mathrm{~N}=1012)=6.59, p=.29\right)$. The study was

conducted over 12 months across 2006-2007. The study was approved by the relevant local ethics committees.

\section{Measures}

Type D Personality Type D was assessed using the DS14 [14]. It is a 14-item measure answered on a five-point Likert type scale ranging from 0 (false) to 4 (true), consisting of two subscales assessing the NA and SI components of Type-D.

Participants who score highly on both NA and SI using a cut-off point of $\geq 10$ on both scales are classified as having a Type D personality: cases [14]. Both subscales were internally consistent in the current study ( $\alpha=.85$ and .82 for NA and SI respectively). Taxometric Analyses

The analysis used taxometric procedures developed by Meehl and colleagues $[13,24-25,27]$ and implemented following recommendations made by Ruscio and colleagues [8, 26, 28-32]. These procedures run over three main steps in terms of (1) identifying valid construct indicators, (2) applying the appropriate taxometric techniques and (3) interpretation [30-32].

Identifying valid indicators: Indicators are retained if they showed good indicator validity, distinguishing Type-D cases (termed taxons) from non-cases (termed complements). Meehl [27] has suggested that valid indicators should have a mean separation expressed in standard units in terms of a Cohen's $d$ of 1.25. Second, of the valid indictors those with high item-total correlations are retained as they represent the most valid indicators of the construct [26]. Finally, evidence for nuisance covariance in the retained indicators is explored. For nuisance covariance to be tolerable, this should be .30 or less [27]. 
Taxometrics of Type-D

Taxometric Techniques: The basic principle of Meehl's taxomeric technique is termed 'coherent cut kinetics' where indictor variables are split into input and output variables. For successive divisions along the input variable either mean differences either side of a cut (mean above minus below a cut: MAMBAC), or co-variances (Maximum Covariance: MAXCOV) or eigenvalues (Maximum Eigenvalues: MAXEIG) within a cut (or sub-sample) are computed for the remaining indictors (called output variables) [25, 30-32]. Based on the General Covariance Mixture Theorem (GCMT) $[25,30]$ the mean difference, covariances or eigenvalues for valid indicators will be at a maximum when the sample contains equal proportions of both taxons and complements and at a minimum when either complement or taxons are present alone. With divisions represented on the $\mathrm{x}$-axis and mean differences, covariance or eigenvalues on the y-axis, characteristic curves represent either a taxonic or dimensional solution. If the latent structure is taxonic (the sample is a mixture of taxons and complements) the curve will be characteristically peaked for MAMBAC (identifying the division that maximally separates complement from taxon) or either peaked or cusped for MAXCOV/MAXEIG ${ }^{2}$. If the structure is dimensional (i.e., there is not a specific taxon) then at different cuts/sub-populations the values should remain virtually unchanged and the graph is either flat or concave.

Which taxometric technique to use depends, in part, on the number of indicators [30]. MAMBAC requires a minimum of two indicators, one is designated the input and one the output. The mean difference on the output variable is calculated above and below successive cuts on the input variable. MAXCOV is used with at least three indicators. One indicator acts as an input variable and is used to divide the sample into a succession of overlapping subsamples or windows. The covariance

\footnotetext{
${ }^{2}$ A MAXCOV/MAXEIG curve is cusped if there are too few number of either taxon or complement subgroups either side of the most heterogeneous subsample (see 8, and 31 for a more detail).
} 
Taxometrics of Type-D between the other output indicators is calculated at successive windows. All possible combinations of input-output triplets are used. MAXEIG is used when there are more than 3 indicators, this is an extension of MAXCOV, where the $1^{\text {st }}$ eigenvalue is calculated across successive windows on the remaining output variables. An alternative approach to 'coherent cut kinetic' is to base taxometric analyses on factor analytic procedures [25]. For example, L-Mode factor analysis requires multiple indicators and uses factor analytic procedures to calculate scores on the $1^{\text {st }}$ principal component and the distribution of these is plotted. A uni-modal distribution indicates a dimensional solution and a bi-modal distribution a taxonic solution.

Interpretation: Interpretation of taxometric analysis involves inspecting the characteristic shape of the curves. As visual inspection can be open to judgement errors and skewed indicators can lead to the erroneous identification of taxa [29-32], Rusico and colleagues have developed a set of interpretative techniques based on comparisons to simulated taxonic and dimensional curves and the development of the curve comparison fit index (CCFI) [30-32]. This approach is based on generating a series of simulated taxonic and dimensional curves (based on 10 replications) derived from the actual data characteristics (see 30 for detail of the simulation algorithm). The actual data is plotted relative to the simulated curves plotted \pm 1 SD. This allows a contextualized interpretation. To further aid interpretation the CCFI, a relative fit index, is used [8,30-32]. The CCFI varies between 0 and 1 and is symmetric around .5. Values greater than .5 indicate a taxonic solution and below .5 a dimensional solution. Extensive Monte-Carlo studies support the precision of these methods relative to all other procedures $[30,32]$. Finally the results need to show consistency. This means using a number of taxometric procedures to show consistency across 
Taxometrics of Type-D

methods and showing the same results while successively increasing the number of cuts/windows [30, 32].

\section{Results}

\section{Prevalence of Type-D}

Using the recommended cutoff point of $\geq 10$ on both NA and SI to define Type-D [14], 39.6\% of females and $34.7 \%$ of males were categorized as Type-D. Type-D classification did not vary as a function of either $\operatorname{sex}\left(\chi^{2}(1)=1.83, p=.41\right)$ or sample region $\left(\chi^{2}(6)=16.32, p=.33\right)$. As Type-D is hypothesized to be an independent risk factor for cardiovascular disease, this prevalence rate appears high and in fact highlights how the simple taxonic model can be somewhat problematic as a prognostic risk factor in a healthy population.

\section{Selection of Indicators}

The indicator validities (expressed in standard units as Cohen's $d$ ), item-total correlations and skew for each indicator are presented in Table 1.

Initially these statistics were examined for the 14 items of the DS14. All indicators showed a degree of skew that was generally within the ranges reported for other taxometric studies [29]. However, only three items, all SI items, had indicator validities equal to or greater than 1.25. Thus at the item level there was not sufficient taxon-complement separation to conduct taxometric analyses.

Examining the two main sub-components of Type-D (i.e., NA and SI) indicated that both showed good indicator validity, had acceptable skew and were correlated with each other $.42, \mathrm{p}<.001$. There is some evidence that NA and SI may themselves be multi-dimensional factors [11]. Principal components analysis was applied separately to the NA and SI items. The results showed that the NA items 
Taxometrics of Type-D

loaded on a single factor (eigenvalue $=3.819, \%$ variance $=54.5 \%$ ). The SI items loaded onto two separate factors (eigenvalues $=3.466 \& 1.004 ; \%$ variance $=63.8 \%$ ). The varimax rotated solution for the SI items are presented in Table 2. The first factor represents being closed and keeping others at a distance and we refer to this primarily as a reticence factor. The second factor represents finding social contact inhibiting and uncomfortable and we refer to this as a social discomfort factor. These three factors (NA, reticence and social discomfort) all showed good indicator validity, itemtotal correlations and acceptable skew. Based on these analyses MAMBAC was applied to NA and SI and MAXCOV to the NA, reticence and social discomfort. With a maximum of 3 indicators there are not a sufficient number to justify MAXEIG or LMode factor analysis. MAMBAC analyses were performed with 50, 150 and 200 cuts and MAXCOV with 50, 150 and 200 windows with 90\% overlap.

Within the putative taxon and complement (using the recommended cutoff point of $\geq 10$ on both NA and SI) the nuisance covariance for NA and SI for the taxon is .30 and for the complement it is -.09 . For the three indicators the mean nuisance covariance for the taxon is .22 and .09 for the complement. Furthermore, nuisance covariance for the 2 indicators in the MAMBAC analysis for the taxon and complement at 50 cuts was -.14 and -.06 respectively; at 150 cuts they were -.15 and .06 respectively and for 200 they were -.15 and -.06 respectively. Similarly mean nuisance covariance for the 3 indicators in the MAXCOV analyses for the taxon and complement at 50 windows was .23 and .26 respectively; at 150 windows they were .23 and .20 and for 200 windows they were .11 and .24 respectively. As such there are no problems with nuisance covariance. 


\section{Taxometric Analyses}

For the MAMBAC analyses the CCFIs were $.32, .32$ and .35 for 50,150 and 200 cuts respectively and for the MAXCOV the CCFIs were $.21, .35$ and .36 for 50 , 150 and 200 windows respectively. All the CCFI values are below .50 suggesting a dimensional interpretation of the latent dimensional structure of Type-D is a more accurate representation than a taxonic one. Confirming this, the taxometric curves for the MAMBAC and MAXCOV analyses all indicated that the actual data were more similar to the simulated dimensional rather than the simulated taxonic data curves. Figure 1 provides the MAXCOV taxometric curves at 50 windows as an example. ${ }^{3}$ **********INSERT FIGURE 1 ABOUT HERE**********

\section{Discussion}

The pattern of results reported here indicates clearly that Type-D is better represented as a dimensional rather than a categorical construct. This finding has important theoretical and clinical implications for Type-D. Theoretical models should focus on additive multi-causal agents or risk factors [8]. Indeed, this is the case for research into Type-D, where a wide variety of mechanisms associated with Type-D have been examined $[4-5,18,20]$. However, more research is now needed to explore the additive nature of these different mechanisms. Thus while it is implicitly used as a taxonic construct, the research agenda supporting Type-D conforms more to one defined for a dimensional construct.

\section{Type-D and a Dimensional Construct in Research and Practice}

Based on the above, theoretical models and clinical interventions should be examined using regression approaches with large unselected samples [8] which also have the advantage of increasing statistical power [33]. One of the main assumptions

\footnotetext{
${ }^{3}$ The remaining MAMBAC and MAXCOV curves are available form the $1^{\text {st }}$ author on request.
} 
Taxometrics of Type-D of Type-D is that it is defined synergistically with ill effects only seen for high NA in combination with high SI; when either trait is low there are no effects on health. Given the dimensional nature of Type-D these potential synergistic effects of Type-D should be tested using both additive and multiplicative regression terms [see 18]. If the same prognostic power for Type-D is not seen when treated as the interaction between these continuous traits then its construct validity could be questioned.

How should Type-D be used in clinical practice as a dimensional rather than a taxonic construct? It has been argued that it is appropriate to draw distinctions within a latent dimensional construct as long as these are systematic and empirically justifiable [8]. This approach is similar to the use of continuous diagnostic signs in physical medicine (e.g., blood pressure, heart rate, temperature) that are integrated to reach a final differential diagnosis. One way to achieve this with a dimensional construct is to identify 'inflection points' [34]. An inflection point expresses the association between the continuous dimensional construct and other relevant clinical data (e.g., bio-markers, prognostic clinical outcomes or treatment responses). The point of inflection marks the position on the continuum where these show dramatic accelerated changes [34]. For example, with Type-D this may indicate the point where there is an increase in cardiovascular mortality. However, the issue for Type-D concerns its definition along two constructs. It may be, therefore, that inflection points need to be either (1) identified for both separately and then these combined or (2) combine the two scales and identify a single infection point or (3) use interaction terms specified with different sliding cuts to identify the point at which the interaction best predicts future prognostic outcomes. With respect to diagnosis it should also be noted that Type-D is a risk factor and not a clinical diagnosis in itself and should be 
Taxometrics of Type-D

used in conjunction with other information. Therefore, the issue of cutoffs is perhaps less crucial rather than defining people with respect to the 'normal' range of scores.

\section{Taxometrics and Psychosomatic Medicine: Functional Syndromes and Bio- markers}

It has been strongly argued that taxometric approaches have implications for many fields beyond their current application mainly to investigating psychopathology (e.g., depression, personality disorder) [15]. Within the field of psychosomatic medicine these may be applied initially to the dimensionality of traits directly relevant to psychosomatic medicine. For example, health anxiety, alexithymia, anxiety and post traumatic stress disorder have all already been shown to be dimensional $[8,21$, 35], whereas Type-A and self-monitoring identified as taxonic [8]. Future work could examine the dimensionality of functional syndromes. This would allow key questions to be answered, such as whether or not medically unexplained/functional syndromes such as irritable bowel syndrome (IBS), chronic fatigue syndrome (CFS) and Gulf War Syndrome are taxonic representing unique syndromes? [36]. Indeed, taxometric analyses could, and should, be applied to existing symptom databases to address these fundamental and important questions. Taxometric procedures could also prove useful with other data types relevant to psychosomatic medicine such as biological markers [37] and social interactions [38]. For example, is there a particular taxon for cortisol under- or over-reactors? Are there people who are able to cope with social stress in a qualitatively different way? Once evidence on the dimensionality (boundaries within a disorder: taxon versus complement) has been identified, issues pertaining to the 
Taxometrics of Type-D

boundaries between syndromes (if CFS and IBS are identified as taxonic are they

distinct or do they overlap?) can be examined [28].

\section{Caveats}

The use of a convenience sample of healthy young adults may lead to questions about the generalizability of the findings. However, the question addressed herein is about the latent-dimensional structure of Type D: if the construct is a general personality trait then its latent structure should be stable across populations. Indeed Denollet [14] used a healthy non-clinical sample as part of the development of the DS14. Furthermore, using a sample that is selected to combine clinical cases (with cardiovascular disease) with healthy non-clinical cases may lead to the identification of a pseudo-taxon [8]. That is, evidence shows that the prevalence of Type-D cases is significantly higher in cardiovascular clinical groups compared to healthy controls [14]. This increased prevalence of Type-D cases in clinical samples may lead to the identification of a pseudo-taxon if combined with a healthy sample where the prevalence rate is significantly lower. However, given that the majority of research in the area of Type-D has been conducted on clinical cardiac samples it is important that the results of these taxometric analyses are replicated in (1) an appropriately sampled clinical sample of cardiovascular disease respondents and (2) a larger healthy community sample without cardiovascular disease, but not in samples that combine clinical and non-clinical cardiovascular samples.

\section{Conclusions}

The present taxometric analysis indicates that Type-D is better represented as a dimensional construct. Future theorizing and research examining the links between Type-D and cardiovascular disease should consider dimensional approaches in order to move this area of inquiry forward. 


\section{References}

[1] Denollet J, Pedersen SS, Vrints CJ, Conraads VM. Usefulness of type D personality in predicting five year cardiac events above ad beyond current symptoms of stress in patients with coronary heart disease. Am J Cardiol 2006; 97: 970-973.

[2] Denollet, J. Personality and risk of cancer in men with coronary heart disease. Psychol Med 1998; 28:_991-995.

[3] Denollet J, Holmes RVF, Vrints CJ, Conraads VM. Unfavourable outcome of heart transplantation in recipients with type D personality. J Heart Lung Transplant 2007; 26: 152-158

[4] Denollet J, Sts SU, Stroobant N, Rombouts H, Gillebert TC, Brutsaert, DL. Personality as independent predictor of long-term mortality in patients with coronary heart disease. Lancet 1996; 347:417-421.

[5] Schiffer AA, Denollet J, Widdershoven JW, Hendriks EH, Smith ORF. Failure to consult for symptoms of heart failure in patients with a type-D personality. Heart 2007; 93: 814-818.

[6] Martens EJ, Jupper N, Pedersen SS, Aquarius AE, Denollet. Type-D personality is a stable taxonomy in post-MI patients over an 1-=8-month period. J Psychosom Res 2007, 63: 545-550.

[7] Habra ME, Linden W, Anderson JC, Weinberg J. Type d personality is related to cardiovascular and neuroendocrine reactivity to acute stress. J Psychosom Res 2003; 55: 235-245.

[8]. Ruscio J, Haslam N, Ruscio AM. Introduction to the taxometric method: A practical guide: Mahwah, NJ: Lawrence Erlbaum Associates. 2006 
Taxometrics of Type-D

[9]. Widiger TA, Trull TJ. Plate tectonics in the classification of personality disorder. Am Psychol 2007; 62: 71-83.

[10]. Marcus DK, Gurely JR, Marchi MM, Bauer C. Cognitive and perceptual variables in hypochondriasis and health anxiety: A systematic review. Clin Psychol Rev 2007; 27: 127-139.

[11]. Emons H.M, Meijer RR, Denollet J. Negative affectivity and social inhibition in cardiovascular disease: Evaluating type-D personality and assessment using item response theory. J Psychosom Res 2007; 63: 27-39.

[12]. Muthen B. Should substance use disorders be considered as categorical or dimensional? Addiction 2006; 101: 6-16.

[13]. Meehl PE. Clarifications about taxometric method. Appl Prevent Psychol 1999; 8: $165-174$.

[14]. Denollett J. DS14: Standard assessment of negative affectivity, social inhibition, and Type D personality. Psychosom Med 2005; 67: 89-97.

[15]. Beauchaine TP. A brief taxometric primer. J Clin Child Adolesc Psychol 2007; 36: $654-676$.

[16]. Denollet J, Vrints CJ, Conraads VM. Comparing Type D personality and older age as correlates of tumor necrosis factor- $\alpha$ dysregulation in chronic heart failure. Brain, Behav Immun 2008; 22: 736-743.

[17]. Molloy GJ, Perkins-Porras L, Strike PC, Steptoe A. Type-D personality and cortisol in survivors of acute coronary syndrome. Psychosom Med 2008; 70: 863-868.

[18]. Whitehead DL, Perkins-Porras L, Strike PC, Magid K, Steptoe A. Cortisol awakening response is elevated in acute coronary syndrome with type-D personality. J Psychosom Res 2007; 6: 419-425. 
Taxometrics of Type-D

[19]. Williams L, O’Carroll RE, O’Connor RC. Type D personality and cardiac output in response to stress. Psychol Health, in press. 2008.

[20]. Williams L, O'Connor RC, Howard S, Hughes BM, Johnston DW, Hay JL, O'Connor DB, Lewis CA, Ferguson E, Sheeh, N, Grealy MA, O'Carroll RE. Type D personality mechanisms of effect: The role of health-related behaviour and social support. J Psychosom Res, 2008; 64: 63-69.

[21]. Ferguson, E. (2009) A taxometric analysis of health anxiety. Psychol Med

[22]. De Fruyt F, Denollet J. Type D personality; A five-factor model perspective. Psychol Health, 2002; 17: 671-683.

[23]. Perbandt K, Hodapp V, Wendt T, Jordan J. The distressed personality (Type D) - correlates with anger, aggression and hostility. Psychotherapie Psychosomatik Medizinische Psychologie, 2006; 56: 310-317.

[24]. Meehl PE, Yonce LJ. Taxometric analysis I: Detecting taxonicity with two quantitative indicators using means above and below a sliding cut (MAMBAC procedure). Psychol Reps, 1994; 74: 1059-1274.

[25]. Waller N G, Meehl PE. Multivariate taxometric procedures. Thousand Oaks, CA: Sage. 1998

[26] Ruscio J, Ruscio AM. A nontechincal introduction to the taxometric method. Understanding Stats, 2004; 3: 151-194.

[27]. Meehl PE. Bootstrap taxometrics: Solving the classification problem in psychopathology. Am Psychol, 1995; 50: 266-275.

[28] Ruscio J, Ruscio AM. Clarifying boundary issues in psychopathology: The role of taxometrics in a comprehensive program of structural research. J Abnorm Psychol, 2004; 113: 24-38. 
Taxometrics of Type-D

[29]. Ruscio J, Ruscio AM, Keane TM. Using taxometric analysis to distinguish a small latent taxon from a latent dimension with positively skewed indicators: The case of involuntary defeat syndrome. J Abnorm Psychol, 2004; 113: 145154.

[30]. Ruscio J, Ruscio AM, Meron M. Applying the bootstrap to taxometric analysis: Generating empirical sampling distributions to help interpret results. Multivar Behavior Res 2007; 42: 349-386.

[31]. Ruscio J. Taxometric analysis: An empirically grounded approach to implementing the method. Crim Justice Behav 2007; 34: 1588-1622.

[32]. Ruscio J, Marcus DK. Detectng small taxa using simulated comparison data: A reanalysis of Beach, Amir, and Bau's (2005) data. Psychol Assess 2007; 19: $241-46$.

[33]. Cohen J, Cohen P. Applied Multiple Regression/Correlation Analysis for the Behavioral Sciences. Lawrence Erlbaum Associates. London. 1983

[34]. Kessler RC. Epidemiology perspectives for the development of future diagnostic systems. Psychopath 2002; 35 158-161.

[35]. Parker JDA, Keefer KV, Taylor GJ, Bagby RM. Latent structure of the Alexithymia construct: A Taxometric investigation. Psychol Assess 2008; 20: $385-396$

[36]. Ferguson E. Is there a Gulf War syndrome? Lancet, 1999; 353: 1182

[37]. Lenzenweger MF, McLachlan G, Rubin DB. Resolving the latent structure if schizophrenia endophenotypes using expectation=maximization-based finite mixture modelling. J Abnorm Psychol 2007; 116, 16-29.

[38]. Haslam N. Categories of social relationship. Cognition 1994; 53: 59-90. 
Table 1. Indicators Statistics

Taxometrics of Type-D

\begin{tabular}{|c|c|c|c|c|}
\hline Items & Domain & $\begin{array}{c}\text { Item- } \\
\text { total }\end{array}$ & Cohen's d & Skew \\
\hline $\begin{array}{l}\text { 1. I make contact easily when I meet } \\
\text { people }\end{array}$ & SI & .49 & 1.05 & 0.55 \\
\hline $\begin{array}{l}\text { 2. I often make a fuss about } \\
\text { unimportant things }\end{array}$ & NA & .33 & 0.63 & -0.19 \\
\hline 3. I often talk to strangers & SI & .20 & 0.56 & -0.08 \\
\hline 4. I often feel unhappy & NA & .60 & 1.05 & 0.29 \\
\hline 5. I am often irritated & NA & .51 & 0.93 & 0.02 \\
\hline $\begin{array}{l}\text { 6. I often feel inhibited in social } \\
\text { interactions }\end{array}$ & SI & .63 & 1.37 & 0.13 \\
\hline 7. I take a gloomy view of things & NA & .64 & 1.21 & 0.75 \\
\hline 8. I find it hard to start a conversation & SI & .57 & 1.25 & 0.47 \\
\hline 9. I am often in a bad mood & NA & .60 & 1.06 & 0.68 \\
\hline 10. I am a closed kind of person & SI & .52 & 1.26 & 0.38 \\
\hline $\begin{array}{l}\text { 11. I would rather keep other } \\
\text { people at a distance }\end{array}$ & SI & .50 & 1.15 & 0.72 \\
\hline $\begin{array}{l}\text { 12. I often find myself worrying } \\
\text { about something }\end{array}$ & NA & .45 & 0.85 & -0.39 \\
\hline 13. I am often down in the dumps & NA & .68 & 1.23 & 0.62 \\
\hline $\begin{array}{l}\text { 14. When socializing, I don't find the } \\
\text { right things to talk about. }\end{array}$ & SI & .56 & 1.22 & 0.64 \\
\hline \multicolumn{5}{|l|}{ Two Sub-Components } \\
\hline Negative Affect $(\alpha=.85)$ & NA & & 1.48 & 0.24 \\
\hline Social Inhibition $(\alpha=.82)$ & SI & $.41 *$ & 1.91 & 0.31 \\
\hline \multicolumn{5}{|l|}{ Three Indicators } \\
\hline Negative Affect $(\alpha=.85)$ & NA & .41 & 1.48 & 0.24 \\
\hline Reticence $(\alpha=.78)$ & SI & .61 & 1.85 & 0.30 \\
\hline Social Discomfort $(\alpha=.70)$ & SI & .43 & 1.25 & 0.24 \\
\hline
\end{tabular}

Note. $*=$ inter-scale correlation 
Table 2. Rotated Factor Matrix for the Social Inhibition Items

\begin{tabular}{|c|c|c|c|}
\hline Item & $\mathrm{F} 1$ & F2 & Construct \\
\hline 10. I am a closed kind of person & .79 & .15 & reticence \\
\hline 11. I would rather keep other people at a distance & .79 & .00 & reticence \\
\hline $\begin{array}{l}\text { 14. When socializing, I don't find the right things } \\
\text { to talk about }\end{array}$ & .69 & .27 & Social discomfort \\
\hline 6. I often feel inhibited in social interactions & .68 & .31 & Social discomfort \\
\hline 3. I often talk to strangers (r) & -.02 & .87 & Social poise \\
\hline 1. I make contact easily when I meet people (r) & .44 & .68 & Social poise \\
\hline 8. I find it hard to start a conversation & .56 & .57 & Social discomfort \\
\hline$\alpha$ & .78 & .70 & \\
\hline
\end{tabular}

F1 $=$ Reticence and F2 $=$ Social Discomfort 
Taxometrics of Type-D

Figure 1. MAXCOV curves based on 50 windows

Note for Figure 1: Smooth lines mark the simulated curves +1 and -1 SD from the mean simulated data. The dark lines represent the actual data 